OPEN ACCESS

Edited by: Tizhong Shan

Zhejiang University, China

Reviewed by:

Gregory C. Henderson,

Purdue University, United States

J. A. Taylor,

Harvard Medical School

United States

*Correspondence:

Ashraf S. Gorgey

ashraf.gorgey@va.gov

tORCID:

Jacob A. Goldsmith orcid.org/0000-0002-1699-4092

Ashraf S. Gorgey orcid.org/0000-0002-9157-6034

Specialty section:

This article was submitted to Lipid and Fatty Acid Research,

a section of the journal

Frontiers in Physiology

Received: 05 November 2021

Accepted: 10 January 2022

Published: 10 February 2022

Citation:

Goldsmith JA, Lai RE, Garten RS, Chen Q, Lesnefsky EJ, Perera RA and Gorgey AS (2022) Visceral Adiposity,

Inflammation, and Testosterone Predict Skeletal Muscle Mitochondrial Mass and Activity in Chronic Spinal

Cord Injury.

Front. Physiol. 13:809845. doi: 10.3389/fphys.2022.809845

\section{Visceral Adiposity, Inflammation, and Testosterone Predict Skeletal Muscle Mitochondrial Mass and Activity in Chronic Spinal Cord Injury}

\author{
Jacob A. Goldsmith ${ }^{1 \dagger}$, Raymond E. Lai ${ }^{1,2}$, Ryan S. Garten ${ }^{3}$, Qun Chen, \\ Edward J. Lesnefsky ${ }^{4,5}$, Robert A. Perera ${ }^{6}$ and Ashraf S. Gorgey ${ }^{1,2 * t}$
}

Background: Mitochondrial health is an important predictor of several health-related comorbidities including obesity, type 2 diabetes mellitus, and cardiovascular disease. In persons with spinal cord injury (SCl), mitochondrial health has been linked to several important body composition and metabolic parameters. However, the complex interplay of how mitochondrial health is affected has yet to be determined in this population.

Objective: In this study, we examined the contribution of visceral adiposity, inflammatory biomarkers, testosterone and circulating serum growth factors as predictors of mitochondrial health in persons with chronic SCl.

Participants: Thirty-three individuals with chronic SCI $(n=27$ Males, $n=6$ Females, age: $40 \pm 13.26$ years, level of injury: C4-L1, BMI: $23 \pm 5.57$ ) participated in this crosssectional study.

Methods: Visceral adipose tissue (VAT) was measured via magnetic resonance imaging (MRI). After an overnight fast, serum testosterone, inflammatory biomarkers [interleukin 6 (IL-6), tumor necrosis factor alpha (TNF- $\alpha)$, c-reactive protein (CRP)], and anabolic growth factors [insulin-like growth factor 1 (IGF-1), insulin-like growth factor binding protein 3 (IGFBP-3)] were measured. Skeletal muscle biopsies were obtained from the vastus lateralis muscle to measure citrate synthase (CS) and Complex III activity. Regression analyses were used to examine predictors of mitochondrial mass and activity.

Results: CS activity was negatively associated with VAT $\left(r^{2}=0.360, p<0.001\right)$, $\operatorname{CRP}\left(r^{2}=0.168, p=0.047\right)$, and positively associated with testosterone $\left(r^{2}=0.145\right.$, 
$p=0.042)$. Complex III activity was negatively associated with VAT relative to total lean mass (VAT:TLM) $\left(r^{2}=0.169, p=0.033\right)$, trended for $\operatorname{CRP}\left(r^{2}=0.142, p=0.069\right)$, and positively associated with testosterone $\left(r^{2}=0.224, p=0.010\right)$. Multiple regression showed CS activity was significantly associated with VAT $+\operatorname{CRP}\left(r^{2}=0.412, p=0.008\right)$ and VAT + Testosterone $\left(r^{2}=0.433, p=0.001\right)$. Complex III activity was significantly associated with VAT relative to total trunk cross-sectional area (CSA) + CRP (VAT:total trunk CSA + CRP; $\left.r^{2}=0.286, p=0.048\right)$ and VAT + Testosterone $\left(r^{2}=0.277\right.$, $p=0.024)$.

Conclusion: Increased visceral adiposity and associated inflammatory signaling (CRP) along with reduced testosterone levels predict mitochondrial dysfunction following SCI. Specifically, lower VATCSA and higher testosterone levels or lower VAT CSA and lower CRP levels positively predict mitochondrial mass and enzyme activity in persons with chronic $\mathrm{SCl}$. Future research should investigate the efficacy of diet, exercise, and potentially testosterone replacement therapy on enhancing mitochondrial health in chronic SCl.

Clinical Trial Registration: [www.ClinicalTrials.gov], identifier: [NCT02660073].

Keywords: spinal cord injury, visceral adipose tissue, mitochondria, inflammation, growth factors, testosterone

\section{INTRODUCTION}

Cardiometabolic disorders are a leading cause of mortality among persons with spinal cord injury (SCI) (Schladen and Groah, 2014). Recent guidelines have emerged to highlight the magnitude of the problem and provide assessment tools for researchers and clinicians to distinguish those at risk (Nash and Gater, 2020). Cardiometabolic syndrome presents as a cluster of disorders, including impaired glucose tolerance, insulin resistance, dyslipidemia, central obesity, and elevated blood pressure (Després and Lamarche, 1993; Després et al., 2001; Grundy et al., 2004). Cardiometabolic syndrome impacts more than 50\% of persons with SCI (LaVela et al., 2006; Dopier Nelson et al., 2007). Today, the root of the problem remains unresolved and likely to continue without appropriate intervention.

Recent emerging cross-sectional studies have clearly associated central obesity with cardiometabolic disorder after SCI. Earlier studies indicated the link between waist circumference and cardiometabolic diseases in persons with SCI (Buchholz and Bugaresti, 2005; Gill et al., 2020). It appears that waist circumference reflects increased visceral adiposity. Gorgey and Gater (2011) were among the first to show that with increasing visceral adipose tissue (VAT), persons with SCI may suffer from impaired glucose intolerance, insulin resistance, and dyslipidemia. Another study showed that people with SCI had 58\% greater VAT than waist circumference matched controls (Edwards et al., 2008). Using a simple linear regression model, Sumrell et al. (2018) showed that a waist circumference greater than $86.5 \mathrm{~cm}$ is equivalent to VAT equal

Abbreviations: SCI, spinal cord injury; LOI, level of injury; MRI, magnetic resonance imaging; SAT, subcutaneous adipose tissue; VAT, visceral adipose tissue; VAT:SAT, ratio of VAT to SAT; LM, Lean mass; TLM, total lean mass; IL-6, interleukin-6; IGF-1, insulin-like growth factor 1; IGFBP-3, insulin-like growth factor binding protein 3; CRP, c-reactive protein; CS, citrate synthase; TNF- $\alpha$, tumor necrosis factor alpha. to or greater than $100 \mathrm{~cm}^{2}$. The same work demonstrated that VAT $>100 \mathrm{~cm}^{2}$ was associated with decreased insulin sensitivity, increased inflammatory biomarkers, and reduced oxygen uptake. In a follow-up trial, the same research group showed that a waist circumference of $86.5 \mathrm{~cm}$ distinguishes those at risk of developing cardiometabolic disorders in persons with SCI (Gill et al., 2020). A recent review summarized potential mechanisms for increasing VAT to contribute to the prevalence of cardiometabolic diseases after SCI (Goldsmith et al., 2021).

Previous work has alluded to several potential mechanisms that likely trigger VAT-associated cardiometabolic disorders (Seidell et al., 1990; Nicklas et al., 2003; Fox et al., 2007; Katzmarzyk et al., 2013; Lee et al., 2018; Gorgey et al., 2021). Although most studies have not demonstrated causality, these studies established potential mechanisms that warrant further investigation. Farkas et al. (2018) and Farkas and Gater (2018) noted that with increasing VAT, there is a potential increase in inflammatory biomarkers, mainly tumor necrosis factor-alpha (TNF- $\alpha$ ) and interleukin-6 (IL-6). Abilmona et al. (2019) noted that, in persons with SCI, hypogonadal individuals $(<300 \mathrm{ng} / \mathrm{dL})$ are likely to have greater VAT than persons with normal testosterone levels. Finally, O'Brien et al. (2017b) described an association between increasing VAT and mitochondrial dysfunction in persons with SCI.

Mitochondrial health is an important predictor of several health-related comorbidities, including obesity, type 2 diabetes mellitus and cardiovascular disease (Poznyak et al., 2020). Mitochondrial function is linked to several important body composition and metabolic parameters in persons with SCI. Specifically, mitochondrial mass (citrate synthase activity) and Complex III activity have been negatively associated with increased body fat and reduced cardiometabolic health (O'Brien et al., 2017a,b, 2018). Based on the above evidence, it appears 
that VAT exerts deleterious cardiometabolic effects in persons with SCI, by increasing systemic inflammation and diminishing testosterone levels that lead to mitochondrial dysfunction. This cross-sectional study aimed to examine the contribution of visceral adiposity, inflammatory biomarkers, testosterone, and circulating serum growth factors as predictors of mitochondrial health in persons with chronic SCI.

\section{MATERIALS AND METHODS}

\section{Participants}

Thirty-three individuals with chronic SCI (age: $40 \pm 13.26$ years, level of injury: C5-L1, BMI: $23 \pm 5.57$ ) participated in this crosssectional study (registered at clinicaltrials.gov: NCT02660073). Only cross-sectional baseline data are presented in this manuscript. All procedures were in accordance with the ethical standards of the Helsinki Declaration of 1,964 and its later amendments. The McGuire Veteran Affairs Investigation Research Board and the Virginia Commonwealth University (VCU) Office of Research and Innovation approved the current study. A neurological examination was performed per the International Standards for Neurological Classification of SCI (ISNCSCI) to determine the American Spinal Injury Association (ASIA) Impairment Scale (AIS) for each participant. Participants provided written, informed consent before the study commenced. Participants with the following pre-existing medical conditions were excluded: active urinary tract infection, those using insulin, hematocrit $>50 \%$, stage 3 pressure sore or above, uncontrolled hypertension, cardiovascular disease or uncontrolled type 2 diabetes mellitus, and individuals with neck of femur or total body osteoporosis (T-score $\leq-2.5$ according to the World health organization guidelines) (Reginster and Burlet, 2006; Gorgey et al., 2019a). All participants were instructed to abstain from exercise, alcohol, and caffeine consumption $24 \mathrm{~h}$ before the examination. Participants underwent a general physical examination to rule out any preexisting cardiac problems that included measuring vital signs and a resting 12lead electrocardiogram. After a 10-12 h fast, a cannula was inserted into an antecubital vein of one arm for blood sampling. Fasting whole-blood samples were drawn into serum separator and potassium oxalate/sodium fluoride tubes and centrifuged to collect serum and plasma samples, respectively. The majority of blood samples were sent to the Chemistry Pathology Laboratory for analysis; however, a subset was sent to a research lab at VCU for further analysis using the same protocol and assay kits. Inflammatory biomarkers (Tumor necrosis factor-alpha; TNF$\alpha$, Interleukin-6; IL-6, and c-reactive protein; CRP) were also analyzed in serum samples by enzyme-linked immunosorbent assays (ELISA) (ALPACO; Salem, NH). Total serum testosterone was measured by liquid chromatography with isotope dilution mass spectrometry detection after supported liquid extraction (Esoterix, Inc.). Testosterone levels in each sample were calculated from a linear plot generated by purified testosterone standards ranging from 2.5 to $5,000 \mathrm{ng} / \mathrm{dL}$. Serum insulinlike growth factor-1 (IGF-I) and insulin-like growth factorbinding protein 3 (IGFBP-3) concentrations were measured with immunoassays (Quantikine R\&D Systems, Inc., Minneapolis, MN, United States).

\section{Magnetic Resonance Imaging}

Abdominal MRI scans were imaged using a 1.5- or 3 Tesla magnet (General Electric, Waukesha, WI) whole-body scanner, using a fast spin-echo sequence described previously (Gorgey et al., $2019 b$ ). Transverse images (slice thickness of $0.8 \mathrm{~cm}$, inter-slice space of $1.2 \mathrm{~cm}$ ) were captured from the xiphoid process to the femoral heads. Depending on the individual's torso length, approximately $20 \pm 30$ images were obtained. Participants were asked to remain as still as possible during the entirety of the scan. In addition, participants were instructed to hold their breath for approximately $20 \mathrm{~s}$ to prevent respiratory artifacts from altering image quality. Images were sequenced anatomically using Image-J software (National Institute of Health, Bethesda, Maryland) and analyzed using Win Vessel software (Win Vessel 2.0, Ronald Meyer, Michigan State University, East Lansing, MI, United States). Each image was automatically segmented into fat and muscle, with bone and background tissue identified based on its signal intensity. Abdominal adipose tissue was separated into subcutaneous adipose tissue (SAT) and VAT depots. An experienced technician manually identified regions of interest guided by anatomical landmarks. The cross-sectional areas (CSA) of these different compartments were used to derive the VAT:SAT ratio to control for regional adiposity. The total area within the outer border of the trunk represented the total trunk CSA, which

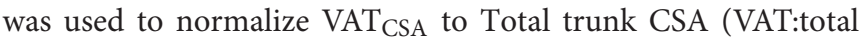
trunk CSA ratio) (Abilmona and Gorgey, 2018). All values were averaged across images to reflect the whole torso.

\section{Dual-Energy X-Ray Absorptiometry}

Total body and regional DXA scans were performed using a GE Lunar iDXA (Lunar Inc., Madison, WI, United States) bone densitometer at the Hunter Holmes VA Medical Center. All scans were performed and analyzed using Lunar software version 10.5. After scanning, total and regional \% fat mass and fat-free mass were determined using DXA software. The longitudinal precision of total and regional body composition using DXA has been determined in persons with SCI (Lester et al., 2019). As previously described, VAT mass was made relative to SAT mass to account for differences in SAT mass between individuals. Additionally, VAT was made relative to total lean mass (TLM) and trunk CSA to account for differences between individuals, as previous research has demonstrated a relationship between VAT mass, total trunk mass, and LM (O'Brien et al., 2017b; Abilmona et al., 2019).

\section{Enzyme Activities}

Muscle biopsies from the vastus lateralis of the right leg were collected using a 14-gauge Tru $\mathrm{Cut}^{\mathrm{TM}}$ needle under local anesthesia (2\% lidocaine). Samples were snap-frozen in liquid nitrogen and stored at $-70^{\circ} \mathrm{C}$. Connective and adipose tissue was removed from a portion of the sample $(\sim 10-$ $25 \mathrm{mg}$ ), then homogenized in ice-cold buffer containing $220 \mathrm{mM}$ mannitol, $70 \mathrm{mM}$ sucrose, $5 \mathrm{mM}$ MOPS, $2 \mathrm{mM}$ EDTA, with cOmplete $^{\mathrm{TM}}$ protease inhibitor cocktail, pH 7.4 (Sigma-Aldrich). 
The homogenate was centrifuged at 2,000 rpm (371 g) for $5 \mathrm{~min}$ at $4^{\circ} \mathrm{C}$ and the supernatant was used for analysis. Protein concentration was quantified, and samples were solubilized in $1 \%$ potassium cholate. Homogenization and assays were completed on the same day. CS and Complex III activity were measured spectrophotometrically in duplicate or triplicate as previously described (Brass et al., 2001; O’Brien et al., 2017a). CS activity, a measure of mitochondrial mass, was measured as the formation of thionitrobenzoate at $412 \mathrm{~nm}$ after the addition of 5, 5dithiobis-(2, 4-nitrobenzoic acid), acetyl-CoA, and oxaloacetate $(n=32)$. Complex III activity was reflected by the rate of cytochrome $c$ reduction in absorbance at $550 \mathrm{~nm}(n=32)$ (Brass et al., 2001; Spinazzi et al., 2012). Antimycin A was used to inhibit Complex III. The activity of Complex III was expressed as the antimycin A-sensitive rate. Absorbance was measured before and after the addition of oxaloacetate, and background absorbance was subtracted from the final reading. Data were converted from arbitrary units per minute to $\mathrm{nmol} / \mathrm{min}$ by using the extinction coefficients of $13.6 \mathrm{mM}^{-1} \mathrm{~cm}^{-1}$ for CS and $19.1 \mathrm{mM}^{-1} \mathrm{~cm}^{-1}$ for Complex III. Data were normalized to $\mathrm{mg}$ of protein added.

\section{Statistical Analysis}

All data were analyzed for normality of distribution. Data that were not normally distributed were log-transformed to permit the use of parametric statistics. Single and multiple linear regression models were used to examine the relationships between measures of mitochondrial mass (citrate synthase activity) and activity (Complex III), visceral adiposity, serum inflammatory biomarkers, anabolic growth factors, and testosterone. We used a maximum of two predictors in each regression to avoid multicollinearity issues due to the relatively low sample size. Instead of adding variables including time since injury (TSI), level of injury (LOI), and age as predictors in these models, we controlled these variables using weighted least squares regressions. We continued with multiple regression models only if a predictor was independently significant with either CS or Complex III activity. Statistical analyses were performed using SPSS (SPSS Statistics version 24, IBM Corp., Armonk, United States). Statistical significance was accepted at a priori of $\alpha \leq 0.05$.

\section{RESULTS}

\section{Participant Characteristics}

Participant demographics and injury characteristics are presented in Table 1. Twenty-two were paraplegic (T1-L1) and 11 were tetraplegic (C4-C8). Participants ranged in age from 20 to 61 and BMI ranged from 14.2 to $35.3 \mathrm{~kg} / \mathrm{m}^{2}$. Age, height, weight, BMI, and TSI were not significantly different between tetraplegics and paraplegics or Caucasians and African Americans. Inflammatory biomarkers, anabolic growth factors, serum testosterone levels, mitochondrial enzyme activity, and MRI outcomes are presented in Table 2. Values were not significantly different between paraplegics and tetraplegics or Caucasians and African Americans. Central adiposity was apparent in 21 and $32 \%$ of the participants using cutoffs of $\geq 100$
TABLE 1 | Baseline demographics and spinal cord injury characteristics for 33 participants.

\begin{tabular}{lc}
\hline Ethnicity & Caucasian: $n=18$ \\
Sex & African American: $n=15$ \\
& Male: $n=27$ \\
Age (year) & Female: $n=6$ \\
Weight (kg) & $40 \pm 13.00$ \\
Height (cm) & $70 \pm 15.18$ \\
BMl (kg/m ${ }^{2}$ ) & $175 \pm 8.99$ \\
Level of injury (range) & $23 \pm 5.25$ \\
Time since injury (yr) & C5-L1 \\
AIS (score) & $11 \pm 10.59$ \\
& A: $n=19$ \\
SCl classification & B: $n=9$ \\
& C: $n=5$
\end{tabular}

BMI, body mass index; AIS, American Spinal Injury Association Impairment Scale; AIS-A, complete motor and sensory loss below the level of injury; AIS-B, complete motor loss and incomplete sensory loss below the level of injury; AIS-C, incomplete motor and sensory loss with less than half of the muscles tested below the LOI graded $\geq 3$. Mean $\pm S D$ unless otherwise noted.

TABLE 2 | Citrate synthase activity, inflammatory biomarkers, anabolic growth factors, and serum testosterone levels.

\begin{tabular}{|c|c|c|c|}
\hline Anabolic growth factors & Mean \pm SD & Range & $\begin{array}{c}\text { Sample } \\
\text { size }\end{array}$ \\
\hline IGF-1 (ng/mL) & $143.99 \pm 58.73$ & 72.90-267.85 & 25 \\
\hline IGFBP-3 (ng/mL) & $1851.40 \pm 368.57$ & 1320.50-2598.55 & 25 \\
\hline \multicolumn{4}{|l|}{ MRI and DXA measures } \\
\hline Total trunk $\mathrm{CSA}_{\mathrm{A}}\left(\mathrm{cm}^{2}\right)$ & $571.44 \pm 174.17$ & $323.16-940.64$ & 28 \\
\hline SAT CSA $\left(\mathrm{cm}^{2}\right)$ & $136.43 \pm 100.30$ & $22.97-369.89$ & 28 \\
\hline $\operatorname{VAT}_{\mathrm{CSA}}\left(\mathrm{cm}^{2}\right)$ & $71.73 \pm 64.97$ & $4.06-220.01$ & 28 \\
\hline VAT:SAT ratio & $0.64 \pm 0.47$ & $0.10-2.22$ & 28 \\
\hline VAT:total trunk CSA & $0.11 \pm 0.08$ & $0.01-0.30$ & 28 \\
\hline VAT:TLM & $0.0015 \pm 0.0013$ & $0.0001-0.0043$ & 28 \\
\hline VAT:Leg LM & $0.0054 \pm 0.0049$ & 0.0004-0.02 & 28 \\
\hline TLM (kg) & $448.48 \pm 77.57$ & 288.83-616.34 & 32 \\
\hline Leg LM (kg) & $131.69 \pm 28.58$ & $64.55-188.77$ & 32 \\
\hline \multicolumn{4}{|l|}{ Inflammatory biomarkers } \\
\hline $\mathrm{CRP}(\mathrm{ng} / \mathrm{mL})$ & $14580.81 \pm 19705.45$ & $185.0-73530.25$ & 25 \\
\hline IL-6 (pg/mL) & $3.18 \pm 2.49$ & $0.68-9.27$ & 22 \\
\hline $\mathrm{TNF}-\alpha(\mathrm{pg} / \mathrm{mL})$ & $22.50 \pm 3.96$ & $15.24-30.63$ & 25 \\
\hline \multicolumn{4}{|l|}{ Serum testosterone levels } \\
\hline Testosterone (ng/dL) & $346.72 \pm 223.71$ & $8.30-751.00$ & 30 \\
\hline \multicolumn{4}{|l|}{ Enzyme activity } \\
\hline $\mathrm{CS}(\mathrm{nmol} / \mathrm{mg} / \mathrm{min})$ & $101.22 \pm 59.83$ & $24.00-303.00$ & 32 \\
\hline Complex III (nmol/mg/min) & $190.00 \pm 125.51$ & $47.10-679.00$ & 32 \\
\hline
\end{tabular}

MRI, magnetic resonance imaging; DXA, Dual energy $x$-ray absorptiometry; SAT, subcutaneous adipose tissue; VAT, visceral adipose tissue; ng/dL, IGF-1, insulinlike growth factor 1; IGFBP-3, insulin-like growth factor binding protein 3; CRP, c-reactive protein; IL-6, interleukin 6; CS, citrate synthase; TNF- $\alpha$, tumor necrosis factor alpha; nanograms per deciliter; CSA, cross sectional area; Kg, kilograms. Mean $\pm S D$ unless otherwise noted.

$\mathrm{cm}^{2}$ MRI VAT $\mathrm{CSA}$, and $\geq 0.66$ VAT:SAT ratio, respectively (Gorgey et al., 2011, 2014). Importantly, a fraction of the samples (eight samples used for IL-6, TNF- $\alpha$, IGF-1, and IGFBP-3) sent to VCU for further analysis experienced degradation that resulted 
in erroneous values. Therefore, we excluded these samples from analyses, which resulted in an uneven sample size depending on the marker be analyzed (Table 2). Figure 1 shows the hypothesized factors predicting mitochondrial health following SCI. This hypothesized model shows that, after SCI, visceral adiposity increases and releases inflammatory cytokines that negatively affect testosterone levels and mitochondrial health. While it is clear that visceral adiposity, inflammation, and testosterone are involved in this deleterious process, there are still factors that remain unidentified.

\section{Independent Predictors of Citrate Synthase Activity}

Figure 2 shows the significant relationships predicting Citrate Synthase (CS) activity. CS activity was negatively associated with VAT $\left(r^{2}=0.360, p<0.001\right), \operatorname{CRP}\left(r^{2}=0.168, p=0.047\right)$, VAT:total trunk CSA $\left(r^{2}=0.372, p<0.001\right)$, and VAT:TLM $\left(r^{2}=0.434\right.$, $p<0.001)$, and positively associated with testosterone $\left(r^{2}=0.145\right.$, $p=0.042$ ) (Table 3). CS activity was not independently associated with VAT:SAT, IL-6, TNF- $\alpha$, IGF-1, or IGFBP-3 (data not shown). The relationships between CS activity and VAT, VAT:total trunk CSA, and VAT:TLM remained significant when controlling for TSI, age, and LOI (Table 4). However, testosterone $\left(r^{2}=0.087\right.$, $p=0.119)$ and CRP $\left(r^{2}=0.081, p=0.176\right)$ were no longer significant when controlling for TSI. When controlling for age or LOI (paraplegic vs. tetraplegic), CRP $\left(r^{2}=0.249, p=0.013\right)$ and testosterone $\left(r^{2}=0.170, p=0.026\right)$ were negatively and positively significant, respectively. Moreover, IL-6 showed a significant negative association with CS activity when controlling for age $\left(r^{2}=0.237, p=0.019\right)$. IL-6 was only a significant predictor of CS activity in participants over $40\left(r^{2}=0.466, p=0.014\right)$ but not participants under $40\left(r^{2}=0.068, p=0.437\right)$.

\section{Multiple Regressions to Predict Citrate Synthase Activity}

CS activity was significantly associated with VAT + CRP $\left(r^{2}=0.412, p=0.008\right)$ and VAT + testosterone $\left(r^{2}=0.433\right.$, $p=0.001)$. Within this model, individuals with lower VAT $\mathrm{CSA}_{\mathrm{A}}$

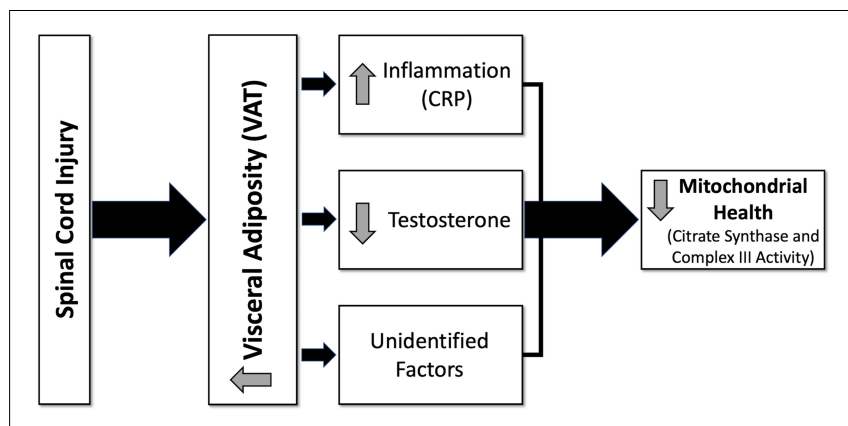

FIGURE 1 | Hypothesized diagram of factors predicting mitochondrial health following spinal cord injury (SCl). After SCl, visceral adiposity increases and releases inflammatory cytokines that negatively affect testosterone levels and mitochondrial health. Visceral adiposity, inflammation, and testosterone are involved in this deleterious process; however, there are still factors that remain unidentified. CRP, c-reactive protein; VAT, visceral adipose tissue. and lower CRP levels had higher CS activity. Furthermore, individuals with lower VAT $\mathrm{CSA}_{\mathrm{A}}$ and higher testosterone levels had higher CS activity. These relationships remained significant when VAT was made relative to total trunk CSA (CRP: $r^{2}=0.454$, $p=0.004$, testosterone: $r^{2}=0.467, p<0.001$ ), and TLM (CRP: $r^{2}=0.444, p=0.005$; testosterone: $\left.r^{2}=0.473, p<0.001\right)$ (Table 3). These relationships also remained significant when controlling for age [VAT + CRP $\left(r^{2}=0.365, p=0.017\right)$, VAT + testosterone $\left.\left(r^{2}=0.382, p=0.004\right)\right]$. As previously stated, IL-6 became significantly negatively associated with CS activity when controlling for age, and VAT + IL-6 $\left(r^{2}=0.412\right.$, $p=0.011$ ) was also significantly negatively associated when controlling for age. Specifically, VAT + IL-6 was a significant predictor of CS activity in participants over $40\left(r^{2}=0.511\right.$, $p=0.041)$ but not in individuals under $40\left(r^{2}=0.293, p=0.353\right)$. Multiple regressions (VAT + CRP, VAT:total trunk CSA + CRP, VAT:TLM + CRP, VAT:total trunk CSA + testosterone, and VAT:TLM + testosterone), remained significant when controlling for TSI, while VAT + testosterone trended toward significance $(p=0.053)$ (Table 4). When controlling for LOI, VAT + CRP $\left(r^{2}=0.503, p=0.002\right)$, VAT + testosterone $\left(r^{2}=0.508\right.$, $p<0.001)$, VAT:total trunk CSA + CRP $\left(r^{2}=0.537\right.$, $p=0.001)$, VAT:total trunk CSA + testosterone $\left(r^{2}=0.534\right.$, $p=<0.001)$, VAT:TLM + CRP $\left(r^{2}=0.531, p=0.001\right)$, and VAT:TLM + testosterone $\left(r^{2}=0.548, p<0.001\right)$ remained significant (Table 4). All other possible models to predict CS activity were tested and not significant.

\section{Independent Predictors of Complex III Activity}

Figure 3 shows the significant relationships predicting Complex III activity. Complex III activity was negatively associated with VAT:TLM $\left(r^{2}=0.169, p=0.033\right)$ but positively associated with testosterone $\left(r^{2}=0.224, p=0.010\right)$. Complex III trended toward significance and was negatively associated with VAT $\left(r^{2}=0.134\right.$, $p=0.061), \operatorname{CRP}\left(r^{2}=0.142, p=0.069\right)$, and VAT:total trunk CSA $\left(r^{2}=0.140, p=0.055\right)$ (Table 5). Complex III activity was not independently associated with VAT:SAT, IL-6, TNF- $\alpha$, IGF-1, or IGFBP-3 (data not shown). Testosterone $\left(r^{2}=0.177, p=0.023\right)$ and VAT:TLM $\left(r^{2}=0.210, p=0.016\right)$ remained significant when controlling for TSI, but CRP became insignificant. Testosterone $\left(r^{2}=0.269, p=0.004\right)$, IL-6 $\left(r^{2}=0.195, p=0.035\right)$, and VAT:TLM $\left(r^{2}=0.148, p=0.047\right)$ were significant when controlling for age, while VAT and VAT:total trunk CSA were no longer significantly associated with Complex III activity ( $p>0.05$; Table 6). All predictors remained significantly associated with Complex III activity when controlling for LOI, while VAT $\left(r^{2}=0.245\right.$, $p=0.009), \operatorname{CRP}\left(r^{2}=0.204, p=0.027\right)$, and VAT:total trunk CSA $\left(r^{2}=0.239, p=0.010\right)$ became significant when controlling for LOI (Table 6).

\section{Multiple Regressions to Predict Complex III Activity}

Complex III was negatively associated with VAT:total trunk CSA + CRP $\left(r^{2}=0.286, p=0.048\right)$ and trended toward significance for VAT + CRP $\left(r^{2}=0.270, p=0.059\right)$ and 

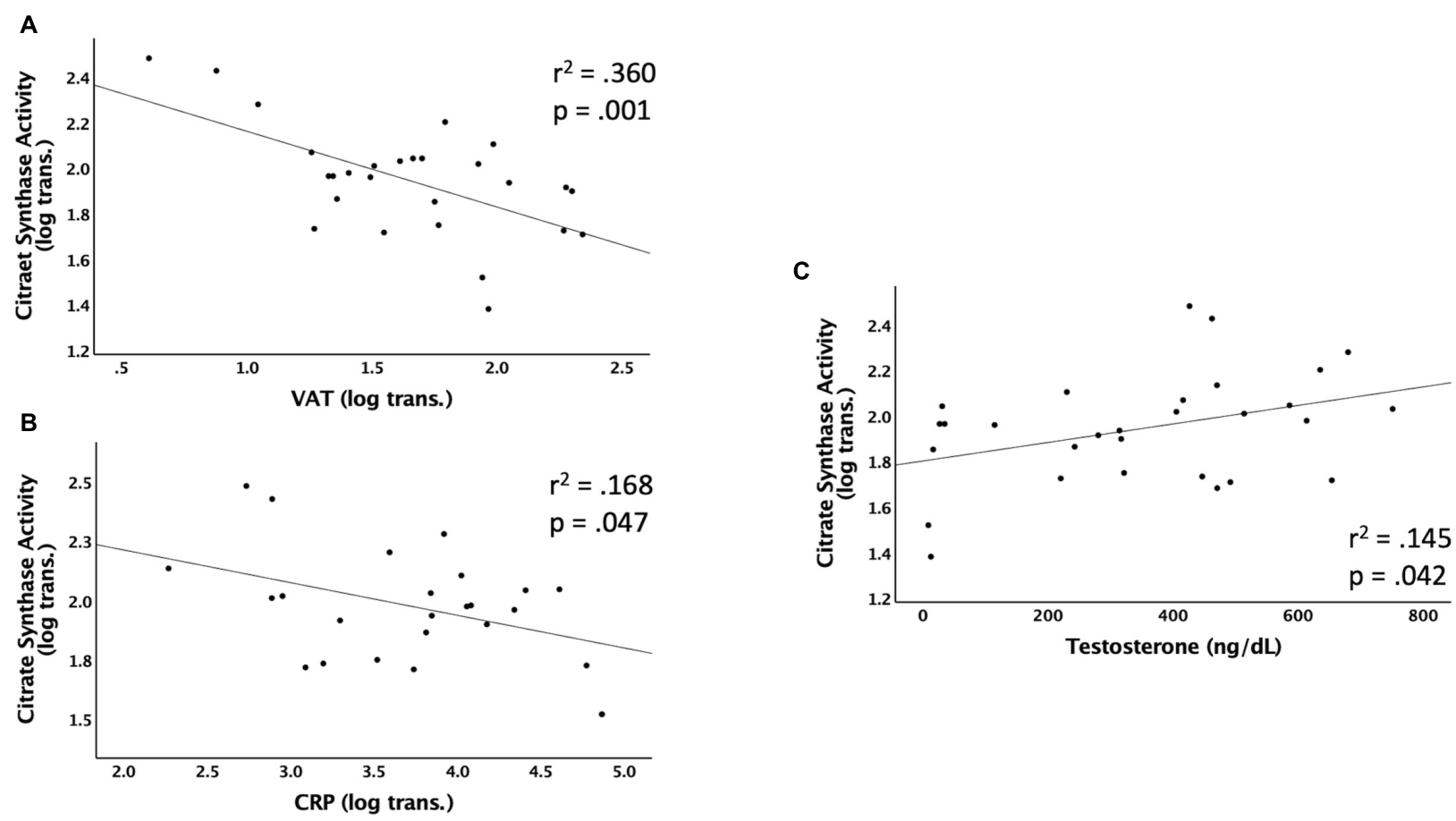

FIGURE 2 | Linear regressions predicting Citrate Synthase (CS) activity. Data that were not normally distributed were log-transformed to permit the use of parametric statistics. The log transformed values of CS enzyme activity are plotted on the $\mathrm{Y}$ axis against the predicted values for each variable on the $\mathrm{X}$ axis. (A) VAT (log transformed) as a predictor of CS activity, (B) CRP (log transformed) as a predictor of CS activity, (C) testosterone (ng/dL) as a predictor of CS activity. VAT, visceral adipose tissue; CRP, c-reactive protein; ng/dL, nanograms per deciliter.

VAT:TLM + CRP $\left(r^{2}=0.275, p=0.055\right)$. Within this model,

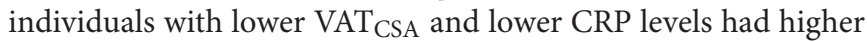
Complex III activity. Complex III was also significantly associated with VAT + testosterone $\left(r^{2}=0.277, p=0.024\right)$, even when VAT was made relative to total trunk CSA $\left(r^{2}=0.303, p=0.016\right)$

TABLE 3 | Single and multiple regressions predicting citrate synthase activity.

\begin{tabular}{lccc}
\hline Predictor variables & $\boldsymbol{\beta}$ & $\boldsymbol{r}^{\mathbf{2}}$ & $\boldsymbol{p}$-value \\
\hline VAT & -0.600 & 0.360 & $<0.001$ \\
Testosterone & 0.380 & 0.145 & 0.042 \\
CRP & -0.410 & 0.168 & 0.047 \\
VAT:total trunk CSA & -0.610 & 0.372 & $<0.001$ \\
VAT:TLM & -0.659 & 0.434 & $<0.001$ \\
VAT + CRP & $-0.530,-0.194$ & 0.412 & 0.008 \\
VAT + Testosterone & $-0.540,0.272$ & 0.433 & 0.001 \\
VAT:total trunk CSA + CRP & $-0.561,-0.211$ & 0.454 & 0.004 \\
VAT:total trunk CSA + Testosterone & $-0.562,0.315$ & 0.467 & $<0.001$ \\
VAT:TLM + CRP & $-0.579,-0.151$ & 0.444 & 0.005 \\
VAT:TLM + Testosterone & $-0.592,0.210$ & 0.473 & $<0.001$ \\
\hline
\end{tabular}

Single and multiple regressions predicting Citrate Synthase activity. Non-significant $r^{2}$ are not shown (non-significant $=n s$ ), but non-significant yet trending $r^{2}$ are included. Standardized Beta weights are presented to demonstrate directionality of associations.

TSI, time since injury; LOI, level of injury; VAT, visceral adipose tissue; TEST, testosterone; CRP, c-reactive protein; IL-6, interleukin 6; LM, lean mass; TLM, total lean mass; CSA, cross sectional area. and TLM $\left(r^{2}=0.286, p=0.021\right)$ (Table 5). Within this model, individuals with lower $\mathrm{VAT}_{\mathrm{CSA}}$ and higher testosterone levels had higher Complex III activity. VAT + testosterone $\left(r^{2}=0.264\right.$, $p=0.029)$ and VAT $+\mathrm{CRP}\left(r^{2}=0.286, p=0.048\right)$ remained significant when controlling for TSI, even when VAT was made relative to total trunk CSA and TLM (Table 6). These relationships remained significant when controlling for age, with the exception of VAT + CRP $\left(r^{2}=0.264, p=0.063\right)$ and VAT:TLM + CRP $\left(r^{2}=0.279, p=0.053\right)$ which trended toward significance (Table 6). When controlling for LOI, VAT + CRP $\left(r^{2}=0.371, p=0.015\right)$ remained significant even when VAT was made relative to total trunk CSA $\left(r^{2}=0.379, p=0.014\right)$ and TLM $\left(r^{2}=0.372, p=0.015\right)$. VAT + testosterone $\left(r^{2}=0.392, p=0.003\right)$ also remained significant when controlling for LOI, even when VAT was made relative to total trunk CSA $\left(r^{2}=0.412, p=0.002\right)$ and TLM $\left(r^{2}=0.397, p=0.003\right)$ (Table 6). Again, individuals with lower VAT $\mathrm{CSA}_{\mathrm{a}}$ and higher testosterone levels had higher Complex III activity within this model. All other possible models to predict Complex III activity were tested and insignificant.

\section{DISCUSSION}

\section{Major Findings}

This study determined mitochondrial health by CS and Complex III activity, surrogate markers for mitochondrial mass, and electron transport chain activity, respectively 
TABLE 4 | Single and multiple regressions predicting Citrate Synthase activity after controlling for TSI, LOI, or age.

\begin{tabular}{|c|c|c|c|c|c|c|}
\hline \multirow[b]{2}{*}{ Marker } & \multicolumn{4}{|c|}{$\begin{array}{l}\text { Citrate synthase activity } \\
\qquad r^{2} \text { (p-value) }\end{array}$} & \multirow[b]{2}{*}{ Age } & \multirow[b]{2}{*}{$\beta$ (Age) } \\
\hline & TSI & $\beta$ (TSI) & LOI & $\beta$ (LOI) & & \\
\hline VAT & $0.174(0.030)$ & -0.418 & $0.432(<0.001)$ & -0.657 & $0.277(0.005)$ & -0.526 \\
\hline Testosterone & ns & 0.296 & $0.170(0.026)$ & 0.412 & $0.176(0.023)$ & 0.420 \\
\hline CRP & ns & -0.285 & $0.249(0.013)$ & -0.499 & $0.184(0.036)$ & -0.429 \\
\hline VAT + CRP & $0.294(0.044)$ & $-0.479,-0.216$ & $0.503(0.002)$ & $-0.546,-0.251$ & $0.365(0.017)$ & $-0.449,-0.273$ \\
\hline VAT + Testosterone & $0.226(0.053)$ & $-0.370,0.238$ & $0.508(0.001)$ & $-0.592,0.279$ & $0.382(0.004)$ & $-0.456,0.334$ \\
\hline VAT:total trunk CSA + CRP & $0.245(0.022)$ & $-0.529,-0.287$ & $0.537(0.001)$ & $-0.571,-0.263$ & $0.415(0.008)$ & $-0.494,-0.298$ \\
\hline VAT:total trunk CSA + Testosterone & $0.250(0.037)$ & $-0.396,0.306$ & $0.534(0.001)$ & $-0.606,0.322$ & $0.414(0.002)$ & $-0.483,0.374$ \\
\hline VAT:TLM + CRP & $0.343(0.023)$ & $-0.533,-0.171$ & $0.531(0.001)$ & $-0.599,-0.200$ & $0.410(0.009)$ & $-0.515,-0.222$ \\
\hline
\end{tabular}

Weighted least squares regressions predicting Citrate Synthase activity when controlling for TSI, LOI, or age. Non-significant ${ }^{2}$ are not shown (non-significant $=n s$ ), but non-significant yet trending $r^{2}$ are included. Standardized Beta weights are presented to demonstrate directionality of associations.

TSI, time since injury; LOI, level of injury; VAT, visceral adipose tissue; CRP, c-reactive protein; IL-6, interleukin 6; LM, lean mass; TLM, total lean mass; CSA, cross sectional area.
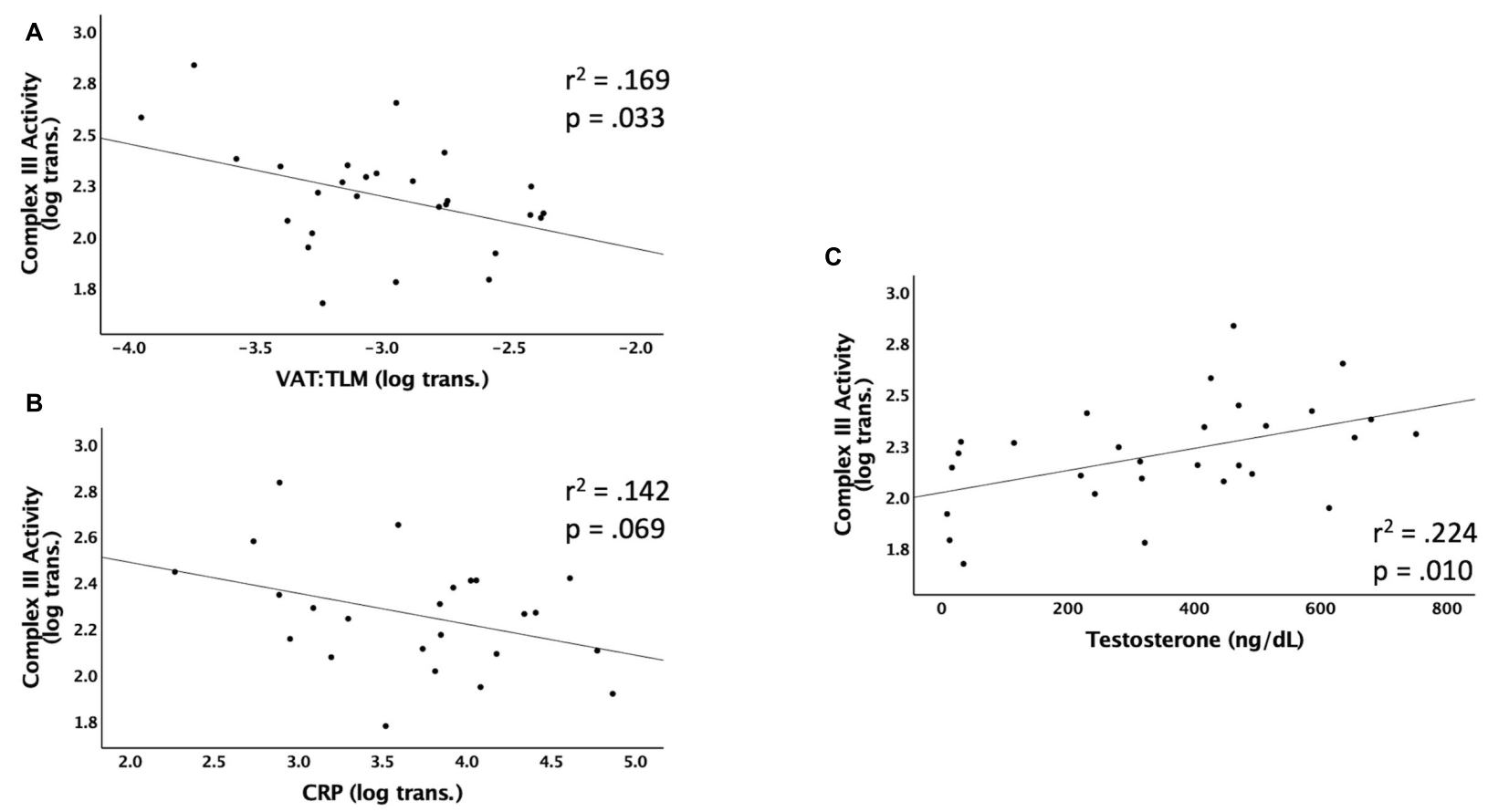

FIGURE 3 | Linear regressions predicting Complex III activity. Data that were not normally distributed were log-transformed to permit the use of parametric statistics. The log transformed values of Complex III enzyme activity are plotted on the $\mathrm{Y}$ axis against the predicted values for each variable on the $\mathrm{X}$ axis. (A) VAT (log transformed) relative to TLM as a predictor of Complex III activity, (B) CRP (log transformed) as a predictor of Complex III activity, (C) testosterone (ng/dL) as a predictor of Complex III activity. VAT, visceral adipose tissue; CRP, c-reactive protein; TLM, total lean mass; ng/dL, nanograms per deciliter.

(O'Brien et al., 2017b). The current findings suggest that reduced mitochondrial mass and enzyme activity can be predicted by increased visceral adiposity, inflammatory signaling, and reduced testosterone levels. Research has demonstrated that inflammation, markers of anabolism, and visceral adiposity are independently associated with mitochondria dysfunction, yet how these factors interact to predict mitochondrial function in chronic SCI is unclear. The results of the current study suggest that, in individuals with chronic SCI, lower VAT $\mathrm{VSA}_{\mathrm{A}}$ and higher testosterone levels or lower VAT $_{\text {CSA }}$ and lower CRP 
TABLE 5 | Single and multiple regressions predicting Complex III activity.

\begin{tabular}{lccc}
\hline Predictor variables & $\boldsymbol{\beta}$ & $\boldsymbol{r}^{\mathbf{2}}$ & $\boldsymbol{p}$-value \\
\hline VAT & -0.366 & 0.134 & 0.061 \\
Testosterone & 0.473 & 0.224 & 0.010 \\
CRP & -0.377 & 0.142 & 0.069 \\
VAT:total trunk CSA & -0.374 & 0.140 & 0.055 \\
VAT:TLM & -0.411 & 0.169 & 0.033 \\
VAT + CRP & $-0.315,-0.294$ & 0.270 & 0.059 \\
VAT + Testosterone & $-0.275,0.390$ & 0.277 & 0.024 \\
VAT:total trunk CSA + CRP & $-0.335,-0.304$ & 0.286 & 0.048 \\
VAT:total trunk CSA + Testosterone & $-0.316,0.408$ & 0.303 & 0.016 \\
VAT:TLM + CRP & $-0.332,-0.275$ & 0.275 & 0.055 \\
VAT:TLM + Testosterone & $-0.299,0.359$ & 0.286 & 0.021
\end{tabular}

Single and multiple regressions predicting Complex III activity. Non-significant $r^{2}$ are not reported, but non-significant yet trending $r^{2}$ are included. Standardized Beta weights are presented to demonstrate directionality of associations.

TSI, time since injury; LOI, level of injury; VAT, visceral adipose tissue; CRP, c-reactive protein; IL-6, interleukin 6; LM, lean mass; TLM, total lean mass; CSA, cross sectional area.

levels positively predict mitochondrial function. The goal of the current study was to elucidate these relationships further to inform future longitudinal intervention programs aimed at enhancing mitochondrial function. The hypothesis that a combination of increased VAT and inflammation along with reduced testosterone levels are associated with the mitochondrial dysfunction seen in chronic SCI was confirmed. To our knowledge, this is the first report to examine the combination of these variables on mitochondrial dysfunction in chronic SCI. Based on the current results, an intervention that reduces visceral adiposity, inflammatory signaling, and optimizes testosterone levels may improve mitochondrial health. Prior work demonstrated that neuromuscular electrical stimulation resistance exercise decreases VAT and inflammatory biomarkers while enhancing citrate synthase and succinate dehydrogenase activities in persons with SCI (Gorgey et al., 2019b, 2020). Future work may examine the effects of exercise or pharmaceutical interventions on the VAT-inflammation-mitochondria axis.

\section{Mitochondrial Function and Visceral Adipose Tissue}

VAT mass was negatively related to both CS and Complex III activity, which agrees with previous results (O'Brien et al., 2017b). The relationships between VAT and CS activity remained significant when controlling for TSI, age, and LOI. VAT was also negatively associated with Complex III activity when VAT was made relative to LM. VAT and VAT:total trunk CSA trended toward significance with Complex III and became significant when controlling for LOI, which may be due to VAT volume being associated with LOI (Farkas et al., 2018). Unlike previous results, VAT:SAT ratio was not independently associated with either CS or Complex III activity (O'Brien et al., 2017b). However, unlike the current study, these previous results were found only in men with motor complete SCI. Overall, there was a clear relationship between visceral adiposity and mitochondrial health. Individuals with lower VAT had higher mitochondrial mass and enzyme activity, which is in agreement with previous research (O'Brien et al., 2017b). Previous research has clearly shown that excess accumulation of VAT appears to play a significant detrimental role in cardiometabolic health (Freedland, 2004; Emmons et al., 2011; Gorgey and Gater, 2011). Indeed, O'Brien et al. (2017) demonstrated that lipid and metabolic profiles are related to mitochondrial mass and activity in individuals with SCI. Similar to the current results, O'Brien et al. (2017b) showed that many body composition measures remained related to CS and Complex III activity when

TABLE 6 | Single and multiple regressions predicting Complex III Activity after controlling for TSI, LOI, or age.

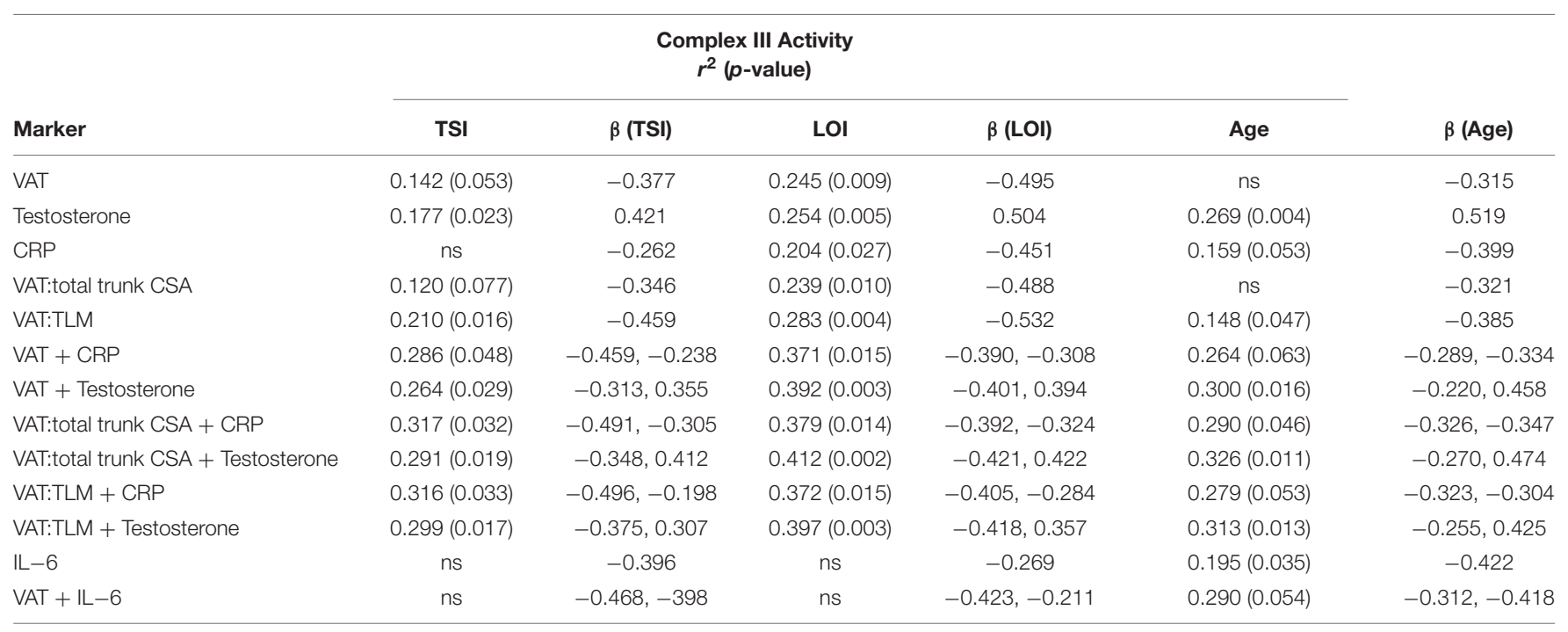

Weighted least squares regressions predicting Complex III activity when controlling for TSI, LOI, or age. Non-significant $r^{2}$ are not shown (non-significant $=$ ns), but non-significant yet trending $r^{2}$ are included. Standardized Beta weights are presented to demonstrate directionality of associations.

TSI, time since injury; LOI, level of injury; VAT, visceral adipose tissue; CRP, c-reactive protein; IL-6, interleukin 6; LM, lean mass; TLM, total lean mass; CSA, cross sectional area. 
normalized to thigh muscle CSA. Together, these results suggest that increased visceral adiposity results in decreased skeletal muscle mitochondrial mass and activity in individuals with chronic SCI. Future interventions targeting both reductions in VAT mass and improved mitochondrial function may enhance cardiometabolic health in individuals with chronic SCI.

\section{Mitochondrial Function and Testosterone}

Testosterone was independently associated with Complex III and CS activity even when controlling for age and LOI. $\mathrm{VAT}+$ testosterone was also significantly associated with both Complex III and CS activity. Individuals with lower VAT CSA $_{\text {A }}$ and higher testosterone levels had higher CS and Complex III activity within these models. These relationships were robust and remained significant when VAT was made relative to total trunk CSA or LM. The levels in the current study were $346.72 \pm 223.7$ $\mathrm{ng} / \mathrm{dL}$, which included six females. When excluding the females, the levels were $428.08 \pm 168.93 \mathrm{ng} / \mathrm{dL}$. Six males (25\%) in the current study were hypogonadal $(<300 \mathrm{ng} / \mathrm{dL}), 11$ males (46\%) were in the low normal range (i.e., 301-500 ng/dL), and seven males $(29 \%)$ were in the upper range of normal $(>500$ $\mathrm{ng} / \mathrm{dL})$. Therefore, the majority of the males in the current study were either hypogonadal or in the low normal range and could potentially benefit from therapeutic replacement. Men with SCI have reduced testosterone levels (Clark et al., 2008; Durga et al., 2011; Bauman et al., 2014; Sullivan et al., 2018), and reduced testosterone levels are associated with greater VAT mass in men with SCI (Abilmona et al., 2019). In persons with SCI, the decline in testosterone levels is $50 \%$ greater than in able-bodied individuals (Gray et al., 1991). VAT is inversely related to plasma total and free testosterone levels in healthy men (Seidell et al., 1990). Deficient testosterone levels increase cardiometabolic risk in young men with chronic SCI. Men with SCI that have total testosterone in the low normal range (i.e., 301-500 $\mathrm{ng} / \mathrm{dL}$ ) have increased risk compared to men with levels in the upper range of normal (>500 ng/dL) (Sullivan et al., 2018). Indeed, low serum testosterone in men with SCI is associated with a poorer cardiometabolic prognosis than men with high serum testosterone (Abilmona et al., 2019). Previous research showed that testosterone replacement therapy can reduce VAT (Gorgey et al., 2019b) and increase peroxisome proliferator-activated receptor- $\gamma$ coactivator- $1 \alpha$ (PGC- $1 \alpha$ ), the master regulator of mitochondrial biogenesis (Gorgey et al., 2020). Future interventions targeting both reductions in VAT mass and optimization of testosterone levels may improve cardiometabolic health in individuals with chronic SCI.

\section{Mitochondrial Function and Inflammation}

CRP was significantly negatively associated with CS activity and trended toward a significant association with Complex III. VAT + CRP was also significantly negatively associated with CS activity. VAT + CRP trended toward a significant association with Complex III and became significant when VAT was made relative to total trunk CSA. Within these models, individuals with lower VAT $_{\text {CSA }}$ and CRP levels had higher CS and Complex III activity. CRP is elevated in persons with chronic SCI (Gibson et al., 2008), is an important predictor of cardiovascular health, and can predict myocardial infarction and stroke (Ridker, 2003). In previous reports, CRP has been associated with VAT mass in various populations (Saijo et al., 2004; Pou et al., 2007; Faber et al., 2010). VAT surrounds the internal organs of the abdominal cavity (Després et al., 2001) and synthesizes and releases proinflammatory cytokines (Yudkin et al., 2005; Farkas and Gater, 2018). VAT secretes higher levels of IL-6, plasminogen activator inhibitor-1, and TNF- $\alpha$ compared to SAT (Farkas and Gater, 2018). While IL-6 and TNF- $\alpha$ were not independently associated with CS or Complex III activity in the current study, CRP is elevated in chronic SCI and is reportedly stimulated by both IL-6 and TNF- $\alpha$ (Edwards et al., 2008; Sam et al., 2009; Farkas and Gater, 2018). However, it is unknown if VAT directly contributes to elevated CRP levels in chronic SCI. Interestingly, IL-6 predicted mitochondrial health, but only in individuals over 40. IL-6 levels have been shown to increase with aging and are associated with increased cardiovascular disease risk (Rea et al., 2018). Future interventions targeting both reductions in VAT mass and inflammation may improve cardiometabolic health in individuals with chronic SCI.

\section{LIMITATIONS}

It should be noted that this study does not precisely identify the complex causal relationships between inflammation, testosterone, anabolic markers, visceral adiposity, and mitochondrial health among those with chronic SCI. This is a correlative analysis based on baseline cross-sectional data. A small number of females ( $n=6$ out of $33, \sim 18 \%$ ) were included in these analyses; however, this may serve as an added benefit to the generalizability of the results of this study because this closely mirrors the actual proportion of males and females living with SCI (NSCISC Annual Report, 2020). This study used a small sample of healthy individuals (i.e., no cardiovascular disease, type 2 diabetes, pressure ulcers, or common medical and psychiatric comorbidities), limiting the generalizability of findings beyond individuals with similar levels of function. Another concern is that conducting simultaneous multiple regressions may result in a multicollinearity problem, especially with a small sample size.

It has been suggested that for a multiple regression with six or more predictors, a sample size of at least 100 is necessary (Green, 1991). In this study, constraints such as access to this specific population and the cost of MRI and DXA limited the sample size. We attempted to limit this multicollinearity problem by including a maximum of two predictors per model. Another limitation is that high variability within the sample resulted in non-normal distributions for several variables. Since we believe it is essential to capture the true variability within the chronic SCI population, we log transformed these variables rather than eliminating statistical outliers, which would have reduced our sample size. For example, removing outliers for VAT alone would have decreased our sample by $\sim 17 \%(n=5)$. The results of this study are exploratory, evaluating potential predictors of mitochondrial function in chronic SCI. Considering these 
limitations, the current findings identify potential predictors of mitochondrial dysfunction following SCI. A large multicenter trial is highly warranted to address the aforementioned limitations effectively.

\section{CONCLUSION}

Increased visceral adiposity, associated inflammatory signaling (CRP), and reduced testosterone levels predict mitochondrial dysfunction following SCI. Specifically, lower VAT CSA $_{\text {and }}$ higher testosterone levels or lower VAT $_{\text {CSA }}$ and lower CRP levels positively predict mitochondrial mass and enzyme activity in persons with chronic SCI (Figure 1). TNF- $\alpha$, IGF-1, and IGFBP-3 were not related to mitochondrial function in this study. IL-6 predicted mitochondrial health, but only in individuals over 40. Future research should further investigate the causal relationships between visceral adiposity, inflammation, testosterone, and mitochondrial health in persons with chronic SCI. Furthermore, interventional studies should be designed to examine the efficacy of diet, exercise, and potentially testosterone replacement therapy on enhancing mitochondrial health in chronic SCI.

\section{DATA AVAILABILITY STATEMENT}

The raw data supporting the conclusions of this article will be made available by the authors, without undue reservation.

\section{ETHICS STATEMENT}

The studies involving human participants were reviewed and approved by the McGuire Veteran Affairs Investigation Research

\section{REFERENCES}

Abilmona, S. M., and Gorgey, A. S. (2018). Associations of the trunk skeletal musculature and dietary intake to biomarkers of cardiometabolic health after spinal cord injury. Clin. Physiol. Funct. Imaging 38, 949-958. doi: 10.1111/cpf. 12505

Abilmona, S. M., Sumrell, R. M., Gill, R. S., Adler, R. A., and Gorgey, A. S. (2019). Serum testosterone levels may influence body composition and cardiometabolic health in men with spinal cord injury. Spinal Cord 57, 229-239. doi: 10.1038/ s41393-018-0207-7

Bauman, W. A., La Fountaine, M. F., and Spungen, A. M. (2014). Age-related prevalence of low testosterone in men with spinal cord injury. J. Spinal Cord Med. 37, 32-39. doi: 10.1179/2045772313Y.0000000122

Brass, E. P., Hiatt, W. R., Gardner, A. W., and Hoppel, C. L. (2001). Decreased NADH dehydrogenase and ubiquinol-cytochrome c oxidoreductase in peripheral arterial disease. Am. J. Physiol. Heart Circ. Physiol. 280, H603-9. doi: 10.1152/ajpheart.2001.280.2.H603

Buchholz, A., and Bugaresti, J. (2005). A review of body mass index and waist circumference as markers of obesity and coronary heart disease risk in persons with chronic spinal cord injury. Spinal Cord 43, 513-518. doi: 10.1038/sj.sc. 3101744

Clark, M. J., Schopp, L. H., Mazurek, M. O., Zaniletti, I., Lammy, A. B., Martin, T. A., et al. (2008). Testosterone levels among men with spinal cord injury: relationship between time since injury and laboratory values.
Board and the Virginia Commonwealth University (VCU) Office of Research and Innovation approved the current study. The patients/participants provided their written informed consent to participate in this study.

\section{AUTHOR CONTRIBUTIONS}

RL, EL, and QC conducted the experiments. RG analyzed blood markers. JG analyzed the data, interpreted the experimental results, designed the figures and tables, and drafted the manuscript. AG, EL, and QC designed the study and interpreted the experimental results. RP assisted with the statistical modeling. AG established funding support. All authors reviewed, revised, and approved the final version of the manuscript.

\section{FUNDING}

This study was supported by the DoD-CDMRP (W81XWH14-SCIRP-CTA). The funding agents have nothing to do with the study design, data collection, analysis, interpretation, or manuscript writing.

\section{ACKNOWLEDGMENTS}

We would like to acknowledge all our study participants, AG, for establishing funding support (DoD-CDMRP, W81XWH-14SCIRP-CTA), the assistance of Refka Khalil, D.C. for research coordination and participant recruitment, Timothy Lavis, M.D., Lance Goetz, M.D., and Teodoro Castillo, M.D. for their help with screenings and physical examinations, technical assistance, and the Hunter Holmes McGuire Medical Center for the opportunity to conduct clinical research.

Am. J. Phys. Med. Rehabil. 87, 758-767. doi: 10.1097/phm.0b013e31818 $37 \mathrm{f} 4 \mathrm{f}$

Després, J.-P., and Lamarche, B. (1993). Effects of diet and physical activity on adiposity and body fat distribution: implications for the prevention of cardiovascular disease. Nutr. Res. Rev. 6, 37-159. doi: 10.1079/NRR199 30010

Després, J.-P., Lemieux, I., and Prud'Homme, D. (2001). Treatment of obesity: need to focus on high risk abdominally obese patients. BMJ 322, 716-720. doi: 10.1136/bmj.322.7288.716

Dopier Nelson, M., Widman, L. M., Ted Abresch, R., Stanhope, K., Havel, P. J., Styne, D. M., et al. (2007). Metabolic syndrome in adolescents with spinal cord dysfunction. J. Spinal Cord Med. 30, S127-S139. doi: 10.1080/10790268.2007. 11754591

Durga, A., Sepahpanah, F., Regozzi, M., Hastings, J., and Crane, D. A. (2011). Prevalence of testosterone deficiency after spinal cord injury. PM R 3, 929-932. doi: 10.1016/j.pmrj.2011.07.008

Edwards, L. A., Bugaresti, J. M., and Buchholz, A. C. (2008). Visceral adipose tissue and the ratio of visceral to subcutaneous adipose tissue are greater in adults with than in those without spinal cord injury, despite matching waist circumferences. Am. J. Clin. Nutr. 87, 600-607. doi: 10.1093/ajcn/87.3.600

Emmons, R. R., Garber, C. E., Cirnigliaro, C. M., Kirshblum, S. C., Spungen, A. M., and Bauman, W. A. (2011). Assessment of measures for abdominal adiposity in persons with spinal cord injury. Ultrasound Med. Biol. 37, 734-741. doi: 10.1016/j.ultrasmedbio.2011.02.002 
Faber, D. R., van der Graaf, Y., Westerink, J., and Visseren, F. L. (2010). Increased visceral adipose tissue mass is associated with increased C-reactive protein in patients with manifest vascular diseases. Atherosclerosis 212, 274-280. doi: 10.1016/j.atherosclerosis.2010.04.029

Farkas, G. J., and Gater, D. R. (2018). Neurogenic obesity and systemic inflammation following spinal cord injury: a review. J. Spinal Cord Med. 41, 378-387. doi: 10.1080/10790268.2017.1357104

Farkas, G. J., Gorgey, A. S., Dolbow, D. R., Berg, A. S., and Gater, D. R. (2018). The influence of level of spinal cord injury on adipose tissue and its relationship to inflammatory adipokines and cardiometabolic profiles. J. Spinal Cord Med. 41, 407-415. doi: 10.1080/10790268.2017.1357918

Fox, C., Massaro, J., Hoffmann, U., Pou, K., Maurovich-Horvat, P., Liu, C., et al. (2007). Abdominal visceral and subcutaneous adipose tissue compartments: association with metabolic risk factors in the Framingham Heart Study. Circulation 116, 39-48. doi: 10.1161/CIRCULATIONAHA.106.67 5355

Freedland, E. S. (2004). Role of a critical visceral adipose tissue threshold (CVATT) in metabolic syndrome: implications for controlling dietary carbohydrates: a review. Nutr. Metab. 1:12. doi: 10.1186/1743-7075-1-12

Gibson, A., Buchholz, A., and Ginis, K. M. (2008). C-Reactive protein in adults with chronic spinal cord injury: increased chronic inflammation in tetraplegia vs paraplegia. Spinal Cord 46, 616-621. doi: 10.1038/sc.2008.32

Gill, S., Sumrell, R. M., Sima, A., Cifu, D. X., and Gorgey, A. S. (2020). Waist circumference cutoff identifying risks of obesity, metabolic syndrome, and cardiovascular disease in men with spinal cord injury. PLoS One 15:e0236752. doi: 10.1371/journal.pone.0236752

Goldsmith, J. A., Ennasr, A. N., Farkas, G. J., Gater, D. R., and Gorgey, A. S. (2021). Role of exercise on visceral adiposity after spinal cord injury: a cardiometabolic risk factor. Eur. J. Appl. Physiol. 121, 2143-2163. doi: 10.1007/s00421-02104688-3

Gorgey, A. S., Dolbow, D. R., Dolbow, J. D., Khalil, R. K., Castillo, C., and Gater, D. R. (2014). Effects of spinal cord injury on body composition and metabolic profile-Part I. J. Spinal Cord Med. 37, 693-702. doi: 10.1179/2045772314Y. 0000000245

Gorgey, A. S., Ennasr, A. N., Farkas, G. J., and Gater, D. R. Jr. (2021). Anthropometric Prediction of Visceral Adiposity in Persons With Spinal Cord Injury. Top. Spinal Cord Inj. Rehabil. 27, 23-35. doi: 10.46292/sci2000055

Gorgey, A. S., and Gater, D. R. (2011). Regional and relative adiposity patterns in relation to carbohydrate and lipid metabolism in men with spinal cord injury. Appl. Physiol. Nutr. Metab. 36, 107-114. doi: 10.1139/H10-091

Gorgey, A. S., Graham, Z. A., Chen, Q., Rivers, J., Adler, R. A., Lesnefsky, E. J., et al. (2020). Sixteen weeks of testosterone with or without evoked resistance training on protein expression, fiber hypertrophy and mitochondrial health after spinal cord injury. J. Appl. Physiol. 128, 1487-1496. doi: 10.1152/japplphysiol.00865. 2019

Gorgey, A. S., Khalil, R. E., Davis, J. C., Carter, W., Gill, R., Rivers, J., et al. (2019a). Skeletal muscle hypertrophy and attenuation of cardio-metabolic risk factors (SHARC) using functional electrical stimulation-lower extremity cycling in persons with spinal cord injury: study protocol for a randomized clinical trial. Trials 20:526. doi: 10.1186/s13063-019-3560-8

Gorgey, A. S., Khalil, R. E., Gill, R., Gater, D. R., Lavis, T. D., Cardozo, C. P., et al. (2019b). Low-dose testosterone and evoked resistance exercise after spinal cord injury on cardio-metabolic risk factors: an open-label randomized clinical trial. J. Neurotrauma 36, 2631-2645. doi: 10.1089/neu.2018.6136

Gorgey, A. S., Mather, K. J., and Gater, D. R. (2011). Central adiposity associations to carbohydrate and lipid metabolism in individuals with complete motor spinal cord injury. Metabolism 60, 843-851. doi: 10.1016/j.metabol.2010. 08.002

Gray, A., Feldman, H. A., McKinlay, J. B., and Longcope, C. (1991). Age, disease, and changing sex hormone levels in middle-aged men: results of the Massachusetts Male Aging Study. J. Clin. Endocrinol. Metab. 73, 1016-1025. doi: $10.1210 /$ jcem-73-5-1016

Green, S. B. (1991). How many subjects does it take to do a regression analysis. Multivariate Behav. Res. 26, 499-510. doi: 10.1207/s15327906mbr2 603_7

Grundy, S. M., Brewer, H. B Jr., Cleeman, J. I., Smith, S. C Jr., Lenfant, C. (2004). Definition of metabolic syndrome: report of the National Heart. Circulation 109, 433-438. doi: 10.1161/01.cir.0000111245.75752.c6
Katzmarzyk, P. T., Heymsfield, S. B., and Bouchard, C. (2013). Clinical utility of visceral adipose tissue for the identification of cardiometabolic risk in white and African American adults. Am. J. Clin. Nutr. 97, 480-486. doi: 10.3945/ajcn.112. 047787

LaVela, S. L., Weaver, F. M., Goldstein, B., Chen, K., Miskevics, S., Rajan, S., et al. (2006). Diabetes mellitus in individuals with spinal cord injury or disorder. J. Spinal Cord Med. 29, 387-395. doi: 10.1080/10790268.2006.11753887

Lee, J. J., Pedley, A., Hoffmann, U., Massaro, J. M., Levy, D., and Long, M. T. (2018). Visceral and intrahepatic fat are associated with cardiometabolic risk factors above other ectopic fat depots: the Framingham Heart Study. Am. J. Med. 131, 684-692. doi: 10.1016/j.amjmed.2018.02.002

Lester, R. M., Ghatas, M. P., Khan, R. M., and Gorgey, A. S. (2019). Prediction of thigh skeletal muscle mass using dual energy $\mathrm{x}$-ray absorptiometry compared to magnetic resonance imaging after spinal cord injury. J. Spinal Cord Med. 42, 622-630. doi: 10.1080/10790268.2019.1570438

Nash, M. S., and Gater, D. R. (2020). Cardiometabolic disease and dysfunction following spinal cord injury: origins and guideline-based countermeasures. Phys. Med. Rehabil. Clin. 31, 415-436. doi: 10.1016/j.pmr.2020.04.005

Nicklas, B. J., Penninx, B. W., Ryan, A. S., Berman, D. M., Lynch, N. A., and Dennis, K. E. (2003). Visceral adipose tissue cutoffs associated with metabolic risk factors for coronary heart disease in women. Diabetes Care 26, 1413-1420. doi: $10.2337 /$ diacare.26.5.1413

NSCISC Annual Report (2020). National Spinal Cord Injury Statistical Center. 2020 Annual Statistical Report for the Spinal Cord Injury Model Systems. Birmingham: University of Alabama at Birmingham.

O'Brien, L. C., Chen, Q., Savas, J., Lesnefsky, E. J., and Gorgey, A. S. (2017). Skeletal muscle mitochondrial mass is linked to lipid and metabolic profile in individuals with spinal cord injury. Eur. J. Appl. Physiol. 117, 2137-2147.

O'Brien, L. C., Wade, R. C., Segal, L., Chen, Q., Savas, J., Lesnefsky, E. J., et al. (2017b). Mitochondrial mass and activity as a function of body composition in individuals with spinal cord injury. Physiol. Rep. 5:e13080. doi: 10.14814/phy2. 13080

O’Brien, L. C., Chen, Q., Savas, J., Lesnefsky, E. J., and Gorgey, A. S. (2017a). Skeletal muscle mitochondrial mass is linked to lipid and metabolic profile in individuals with spinal cord injury. Eur. J. Appl. Physiol. 117, 2137-2147. doi: 10.1007/s00421-017-3687-9

O’Brien, L. C., Graham, Z. A., Chen, Q., Lesnefsky, E. J., Cardozo, C., and Gorgey, A. S. (2018). Plasma adiponectin levels are correlated with body composition, metabolic profiles, and mitochondrial markers in individuals with chronic spinal cord injury. Spinal Cord 56, 863-872. doi: 10.1038/s41393-018-0089-8

Pou, K. M., Massaro, J. M., Hoffmann, U., Vasan, R. S., Maurovich-Horvat, P., Larson, M. G., et al. (2007). Visceral and subcutaneous adipose tissue volumes are cross-sectionally related to markers of inflammation and oxidative stress: the Framingham Heart Study. Circulation 116, 1234-1241. doi: 10.1161/ CIRCULATIONAHA.107.710509

Poznyak, A. V., Ivanova, E. A., Sobenin, I. A., Yet, S.-F., and Orekhov, A. N. (2020). The role of mitochondria in cardiovascular diseases. Biology 9:137.

Rea, I. M., Gibson, D. S., McGilligan, V., McNerlan, S. E., Alexander, H. D., and Ross, O. A. (2018). Age and age-related diseases: role of inflammation triggers and cytokines. Front. Immunol. 9:586. doi: 10.3389/fimmu.2018.00586

Reginster, J.-Y., and Burlet, N. (2006). Osteoporosis: a still increasing prevalence. Bone 38, 4-9. doi: 10.1016/j.bone.2005.11.024

Ridker, P. M. (2003). C-reactive protein: a simple test to help predict risk of heart attack and stroke. Circulation 108, e81-5. doi: 10.1161/01.CIR.0000093381. 57779.67

Saijo, Y., Kiyota, N., Kawasaki, Y., Miyazaki, Y., Kashimura, J., Fukuda, M., et al. (2004). Relationship between C-reactive protein and visceral adipose tissue in healthy Japanese subjects. Diabetes Obes. Metab. 6, 249-258. doi: 10.1111/j. 1462-8902.2003.0342.x

Sam, S., Haffner, S., Davidson, M. H., D’Agostino, R. B., Feinstein, S., Kondos, G., et al. (2009). Relation of abdominal fat depots to systemic markers of inflammation in type 2 diabetes. Diabetes Care 32, 932-937. doi: 10.2337/dc081856

Schladen, M., and Groah, S. (2014). State of the science on cardiometabolic risk after spinal cord injury: recap of the 2013 ASIA pre-conference on cardiometabolic disease. Top. Spinal Cord INJ. Rehabil. 20, 105-112. doi: 10. 1310/sci2002-105

Seidell, J. C., Björntorp, P., Sjöström, L., Kvist, H., and Sannerstedt, R. (1990). Visceral fat accumulation in men is positively associated with insulin, glucose, 
and C-peptide levels, but negatively with testosterone levels. Metabolism 39, 897-901. doi: 10.1016/0026-0495(90)90297-p

Spinazzi, M., Casarin, A., Pertegato, V., Salviati, L., and Angelini, C. (2012). Assessment of mitochondrial respiratory chain enzymatic activities on tissues and cultured cells. Nature protocols 7, 1235-1246. doi: 10.1038/nprot.20 12.058

Sullivan, S. D., Nash, M. S., Tefara, E., Tinsley, E., and Groah, S. (2018). Relationship between gonadal function and cardiometabolic risk in young men with chronic spinal cord injury. PMR 10, 373-381. doi: 10.1016/j.pmrj.2017. 08.404

Sumrell, R. M., Nightingale, T. E., McCauley, L. S., and Gorgey, A. S. (2018). Anthropometric cutoffs and associations with visceral adiposity and metabolic biomarkers after spinal cord injury. PLoS One 13:e0203049. doi: 10.1371/ journal.pone. 0203049

Yudkin, J. S., Eringa, E., and Stehouwer, C. D. (2005). "Vasocrine" signalling from perivascular fat: a mechanism linking insulin resistance to vascular disease. Lancet 365, 1817-1820. doi: 10.1016/S0140-6736(05)66585-3
Conflict of Interest: The authors declare that the research was conducted in the absence of any commercial or financial relationships that could be construed as a potential conflict of interest.

Publisher's Note: All claims expressed in this article are solely those of the authors and do not necessarily represent those of their affiliated organizations, or those of the publisher, the editors and the reviewers. Any product that may be evaluated in this article, or claim that may be made by its manufacturer, is not guaranteed or endorsed by the publisher.

Copyright (๑ 2022 Goldsmith, Lai, Garten, Chen, Lesnefsky, Perera and Gorgey. This is an open-access article distributed under the terms of the Creative Commons Attribution License (CC BY). The use, distribution or reproduction in other forums is permitted, provided the original author $(s)$ and the copyright owner(s) are credited and that the original publication in this journal is cited, in accordance with accepted academic practice. No use, distribution or reproduction is permitted which does not comply with these terms. 